\title{
Spatial and Temporal Distribution of Odorant-Evoked Activity in the Piriform Cortex
}

\author{
Robert L. Rennaker, ${ }^{1}$ Chien-Fu F. Chen, ${ }^{2}$ Andrea M. Ruyle, ${ }^{1}$ Andrew M. Sloan, ${ }^{1}$ and Donald A. Wilson ${ }^{2}$ \\ ${ }^{1}$ School of Aerospace and Mechanical Engineering and ${ }^{2}$ Department of Zoology, University of Oklahoma, Norman, Oklahoma 73019
}

Despite a remarkably precise spatial representation of odorant stimuli in the early stages of olfactory processing, the projections to the olfactory (piriform) cortex are more diffuse and show characteristics of a combinatorial array, with extensive overlap of afferent inputs and widespread intracortical association connections. Furthermore, although there is increasing evidence for the importance of temporal structure in olfactory bulb odorant-evoked output, little is known about how this temporal patterning is translated within cortical neural ensembles. The present study used multichannel electrode arrays and paired single-unit recordings in rat anterior piriform cortex to test several predictions regarding ensemble coding in this system. The results indicate that odorants evoke activity in a spatially scattered ensemble of anterior piriform cortex neurons, and the ensemble activity includes a rich temporal structure. The most pronounced discrimination between different odorants by cortical ensembles occurs during the first inhalation of a $2 \mathrm{~s}$ stimulus. The distributed spatial and temporal structure of cortical activity is present at both global and local scales, with neighboring single units contributing to coding of different odorants and active at different phases of the respiratory cycle. Finally, cross-correlogram analyses suggest that cortical unit activity reflects not only afferent input from the olfactory bulb but also intrinsic activity within the intracortical association fiber system. These results provide direct evidence for predictions stemming from anatomical- and theoretical-based models of piriform cortex.

Key words: microelectrode arrays; olfactory cortex; neural ensembles; cross-correlation; olfaction; odor perception; memory

\section{Introduction}

In most sensory systems, the encoding of information about stimulus identity by neocortical circuits includes both spatial and temporal components. For example, neurons within neocortical columns share response properties, and neighboring columns display more similar response properties than more distant columns. This spatial patterning of tuning properties is primarily driven by localized thalamic afferent input to excitatory and inhibitory cortical neurons (Alonso and Swadlow, 2005), whereas extensive intracortical connections promote intracolumnar synchronization and serve as a substrate for experience-dependent cortical plasticity (Gilbert et al., 2001; Ghose, 2004).

In the mammalian olfactory system, central coding of odor quality information initially also has spatial and temporal components. Different odorants induce odorant-specific spatial patterns of olfactory bulb glomerular layer activity (Leon and Johnson, 2003). Olfactory bulb glomeruli receive input from a homogenous population of olfactory receptor neurons expressing the same olfactory receptor gene (Buck, 1996). Glomeruli serve as the site of synaptic contact between olfactory receptor

Received Sept. 18, 2006; revised Dec. 18, 2006; accepted Jan. 8, 2007.

This work was supported by National Institute on Deafness and Other Communication Disorders Grants R21 DC007112 and R01 DC03906. We thank Drs. Joe Bastian and Maurice Chacron for discussion and advice in early phases of this work and Marilina Resasco for technical assistance in histological analyses.

Correspondence should be addressed to Donald A. Wilson, Department of Zoology, University of Oklahoma, Norman, 0K 73019. E-mail: dwilson@ou.edu.

DOI:10.1523/JNEUROSCI.4072-06.2007

Copyright $\odot 2007$ Society for Neuroscience $\quad$ 0270-6474/07/271534-09\$15.00/0 neurons and second-order neurons, mitral/tufted cells. Most naturally occurring odors are complex mixtures, and the spatial pattern of glomerular activity reflects both individual components (Lin et al., 2006) and early intercomponent interactions (Joerges et al., 1997; Vickers et al., 1998; Tabor et al., 2004). In addition to spatial patterns, both glomerular (Spors et al., 2006) and mitral/ tufted cell activity (Meredith, 1986; Cang and Isaacson, 2003) demonstrate stimulus-specific temporal structure.

The piriform cortex, which receives direct mitral/tufted cell input, is a trilaminar archicortical structure that has an organization consistent with a combinatorial, auto-associative array (Haberly and Bower, 1989; Hasselmo et al., 1990; Haberly, 2001). Thus, rather than displaying a columnar architecture, afferent input from individual olfactory bulb glomeruli terminates in broad patches overlapping with terminal patches of other glomeruli (Zou et al., 2001). This provides an anatomical substrate for convergence between activity of multiple olfactory receptor types (Zou et al., 2005) and therefore promotes the emergence of synthetic processing. The convergence is amplified by an extensive intrinsic association fiber system, wherein a single piriform cortical pyramidal cell may contact several thousand other piriform cortical neurons (Johnson et al., 2000). As in neocortex, this intrinsic association pathway is plastic and has been hypothesized to serve an important role in forming templates of familiar odorants, which facilitates subsequent discrimination and recognition (Haberly, 2001; Wilson and Stevenson, 2003).

Based on piriform cortical architecture, several predictions can be made concerning spatial and temporal odor response pat- 
terns in this structure. First, neurons responsive to particular odorants should be widely distributed throughout piriform cortex, with neighboring neurons potentially displaying different response characteristics. Recent immunohistochemical mapping studies (e.g., c-fos) have supported this conclusion (Illig and Haberly, 2003; Zou et al., 2005), although given the nature of these techniques, information about response dynamics is lost. Thus, it is unknown whether spatial patterns of piriform activation vary over time. In fact, the second prediction is that piriform cortical neurons may show spatiotemporal response complexity, given the dynamics of glomerular (Spors et al., 2006) and mitral/ tufted cell (Meredith, 1986; Mazor and Laurent, 2005) odorant responses in the time range of $0.1-1 \mathrm{~s}$. Third, cortical cell activity may not solely be driven by afferent input but may also reflect intracortical cell-cell interactions. Cross-correlation analyses of simultaneously recorded spike trains can be used to identify such interactions (Perkel et al., 1967; Gerstein and Perkel, 1972; Katz et al., 2002). In this series of studies, we sought initial evidence for these three predictions using microelectrode array and paired single-unit recording techniques.

\section{Materials and Methods}

Subjects. Male Long-Evans hooded rats $(250-500 \mathrm{~g})$ were used as subjects. Animals had food and water available ad libitum and were housed on a $12 \mathrm{hlight/dark} \mathrm{cycle.} \mathrm{Recordings} \mathrm{were} \mathrm{made} \mathrm{during} \mathrm{the} \mathrm{light} \mathrm{phase} \mathrm{of}$ the cycle. All experimental procedures were in accord with Public Health Service guidelines and approved by the University of Oklahoma Institutional Animal Care and Use Committee.

Multielectrode array recordings. The subjects were initially anesthetized using ketamine, xylazine, and acepromazine (targeted dosage of 50, 20, and $5 \mathrm{mg} / \mathrm{kg}$, respectively). Once the subjects were prepped for surgery, they were given an intraperitoneal injection of $1.5 \mathrm{~g} / \mathrm{kg}$ urethane. A thermal regulated heating pad was used to maintain core body temperature. A midline incision was made in the scalp, and the connective tissue was dissected from the skull. A $4 \times 4 \mathrm{~mm}$ section of the left frontal bone adjacent to the lateral ridge and rostral to the coronal suture was removed. An incision was made in the dura exposing the pia. Multichannel electrodes consisted of $25-\mu \mathrm{m}$-diameter microwires (35 $\mu \mathrm{m}$ outer diameter with insulation) with $500 \mu \mathrm{m}$ center-to-center spacing in a $4 \times 2$ matrix (Rennaker et al., 2005). A bone screw was used as reference. The array was initially lowered to a depth of $5.7 \mathrm{~mm}$ from the surface and then gradually advanced until respiratory driven responses were obtained on more than one electrode (Fig. 1). Respiration was monitored with a piezoelectric device strapped to the chest.

Neural (multiunit) data were collected continuously on all channels at $10 \mathrm{kHz}$ sampling rate and bandpass filtered from 500 to $5000 \mathrm{~Hz}$. Spike 2 software (Cambridge Electronics Design, Cambridge, UK) was used to sort multiunit clusters and export the data to Matlab (MathWorks, Natick, MA) for additional analysis. Ten presentations of a series of six odors were presented randomly to each subject with interstimulus intervals of at least $60 \mathrm{~s}$. Spatial and temporal plots of the data were generated by reconstruction of the electrode locations from histology into $100 \mu \mathrm{m}^{2}$ grid two-dimensional plots (see below). A moving window analysis (200 ms) was used to calculate the spike rates on each electrode during spontaneous and odorant-evoked activity. To quantitatively examine differences in the responses to the 10 repetitions of each odorant between different stimuli at each site, paired $t$ tests were used to determine whether responses to the different odorants at each location were significantly different $(\alpha=0.01)$. For group difference plot maps, significant relative increases in activity responses were given a value of 1 , and significant relative decreases in activity were given a value of -1 .

Paired-unit recordings. Rats were anesthetized with urethane (1.5 $\mathrm{g} / \mathrm{kg}$ ), and respiration was monitored via chest wall movements as above. Single-unit recordings were made from anterior piriform cortex with tungsten microelectrodes (5-10 M $\Omega$ ) as described previously (Wilson, 1998; Kadohisa and Wilson, 2006b). These electrodes provided substantially better unit isolation than that depicted in Figure 1 for the array

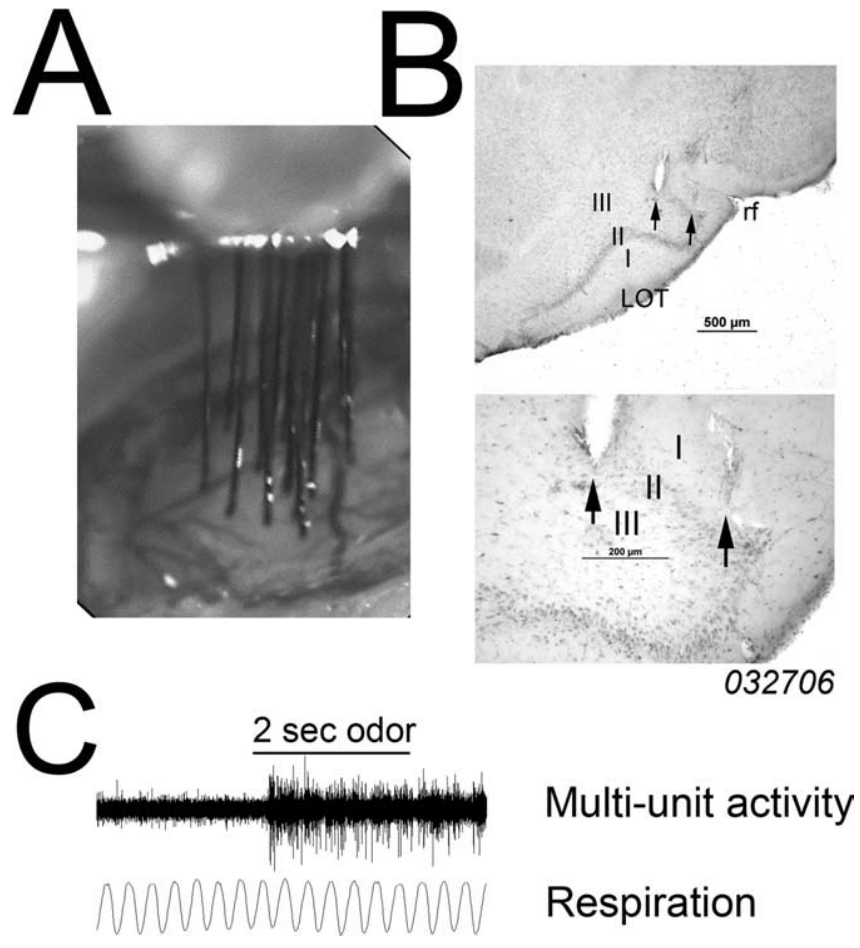

Figure 1. A, Electrode arrays were constructed of six to eight $35 \mu \mathrm{m}$ insulated microwires arranged in two parallel rows and $250 \mu \mathrm{m}$ inter-electrode distances. This is an image of the array placed at the cortical surface. $\boldsymbol{B}$, Recording sites were confirmed to be within layer II/III of anterior piriform cortex histologically. Arrows indicate the location of two electrode tips. LOT, Lateral olfactory tract; if, rhinal fissure. C, Example of multiunit activity recorded from the electrode array and simultaneously recorded respiration (chest wall movement).

electrodes. Layer II/III single units were identified by electrical stimulation of the lateral olfactory tract and subsequent histological confirmation. The lateral olfactory tract was stimulated with a monopolar tungsten electrode (0.1-ms-duration square-wave pulses, $10-300 \mu$ A). Single units were identified off-line using template matching of waveforms with peak amplitudes that exceeded a user-set threshold. Templated waveforms (25-36 data points each) were then subjected to principal component analyses and plotted in three-dimensional space based on the values for the three most significantly weighted factors. Units were then identified by clusters within this space. The single-unit nature of the recordings were confirmed with autocorrelations showing at least a $2 \mathrm{~ms}$ refractory period. All analyses were performed with Spike 2 software (Cambridge Electronics Design). Recordings that allowed at least two single units to be clearly isolated were used here. After isolation of unit activity, spontaneous and odorant-evoked activity ( 2 s odorant pulses, separated by at least $60 \mathrm{~s}$ ) were recorded.

Odorant-evoked activity was determined from cumulative peristimulus time histograms (100 ms bin width) based on 2-10 stimulus repetitions. Significant responses to odorants were determined by comparing binned activity during the stimulus with the mean $\pm 2 \times$ SD of baseline activity. The relationship of single-unit activity to the respiratory cycle was also determined with phase histograms with $6^{\circ}$ bin widths. Activity within the respiratory phase histograms were converted to vectors for determination of mean phase angles.

Finally, cross-correlograms were created with $1 \mathrm{~ms}$ bin width $( \pm 250$ $\mathrm{ms}$ total width) and displayed as both raw spike counts and as smoothed with a 9-ms-wide sliding average. Statistical significance of the peak in the raw (1 ms bin) cross-correlograms was determined in two manners. First, all cross-correlograms were analyzed by $t$ test comparisons between bin counts in the tails of the correlogram (125 ms) with those surrounding the peak $( \pm 25 \mathrm{~ms})$. Given the relatively low firing rate of piriform cortical units compared with many other systems in which data such as this are examined, these time windows were chosen to provide a sufficient sample for robust peak detection without a major loss in temporal 
resolution. In addition, comparisons of crosscorrelograms in some cell pairs were further analyzed with statistical tests as described previously (Abeles, 1982; Bastian and Nguyenkim, 2001) (see Results).

Odorant stimulation. Odorants were presented with a flow-dilution olfactometer with final concentrations at 1:100 to $1: 10$ of saturated vapor. Total flow rate was $1 \mathrm{~L} / \mathrm{min}$, and stimulus durations were $2 \mathrm{~s}$. Odorant onset was triggered on the inhalation/exhalation transition of the respiratory cycle. This transition was chosen to allow stimulus intensity to stabilize in front of the nose during exhalation and before the first inhalation of the stimulus as described previously (Wilson, 1998). Interstimulus intervals were at least $60 \mathrm{~s}$ to avoid cortical adaptation (Wilson, 1998). Odorants included isoamyl acetate, limonene, heptanal, propyl butyrate, benzyl acetate, peppermint, and a homologous series of ethyl esters ranging from ethyl proprionate to ethyl octanoate. Not all animals were tested with all odorants.

Histology. Animals were overdosed with anesthetic (urethane or pentobarbital) and perfused transcardially with saline and $4 \%$ paraformaldehyde, and the brain was subsequently sectioned coronally at $40 \mu \mathrm{m}$ and stained with cresyl violet. In array implanted animals, given that electrodes often diverged from a directly vertical path, the track of each electrode had to be completely reconstructed from the dorsal brain surface to the piriform to allow linking of the electrode recording site with physiological data acquired over that channel. Electrode array recording sites were reconstructed and localized within a three-dimensional grid with the rostral pole of the olfactory tubercle as the anterior zero point, the cerebral midline as the mediolateral zero point, and the ventralmost edge of the brain as the ventrodorsal zero point. This coordinate system allowed composite images to be made across all animals. Recording sites were then flattened along the dorsoventral axis to create two-dimensional images. Individual recording sites and the activity derived from those sites were assigned to a $100 \mu \mathrm{m}^{2}$ grid location for images.

\section{Results}

Electrode array recordings were made in 10 rats with histological confirmation of recording sites within anterior piriform cortex. A total of 59 recording sites with unit activity were obtained from these animals and provide the data described here. In addition, recordings from 39 single-unit pairs were made from 15 different rats.

\section{Distributed responses}

Unit recordings from electrode arrays demonstrated robust and reliable odorant-evoked responses. Individual recording sites responded to multiple odorants, and individual odorants activated units in multiple sites. As shown in Figure 2, individual recording sites reliably responded to molecularly diverse odorants. Individual recording sites showed differential responsiveness to odorants, with vigorous excitation to some odorants and no response or suppression to others. As noted below, the temporal structure of the responses, including latency, duration, and respiratory en-
RAT 209 electrode 3

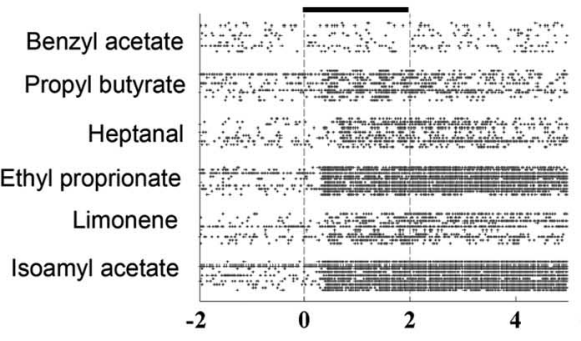

RAT 217 electrode 6

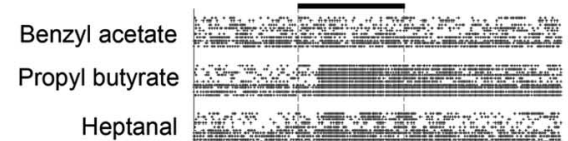

Ethyl proprionate

Limonene

Isoamyl acetate

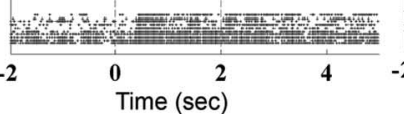

RAT 14 electrode 4

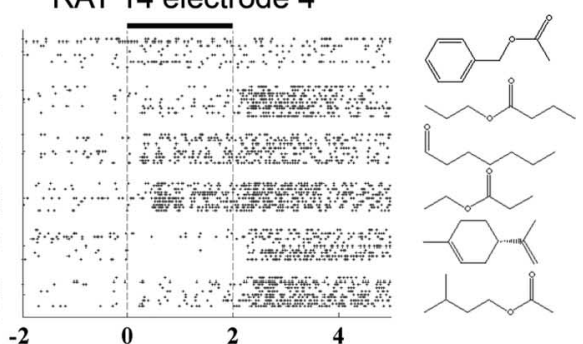

RAT 32 electrode 5
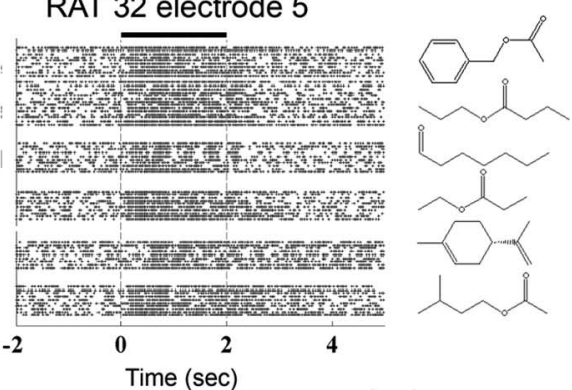

Figure 2. Individual recording sites respond to multiple odorants. Representative raster displays of multiunit activity recorded with electrode arrays are shown. Recordings from single electrodes in four different rats are shown. Each recording site responded to a unique combination of odorants, and responses varied in both magnitude of firing rate and in temporal structure.
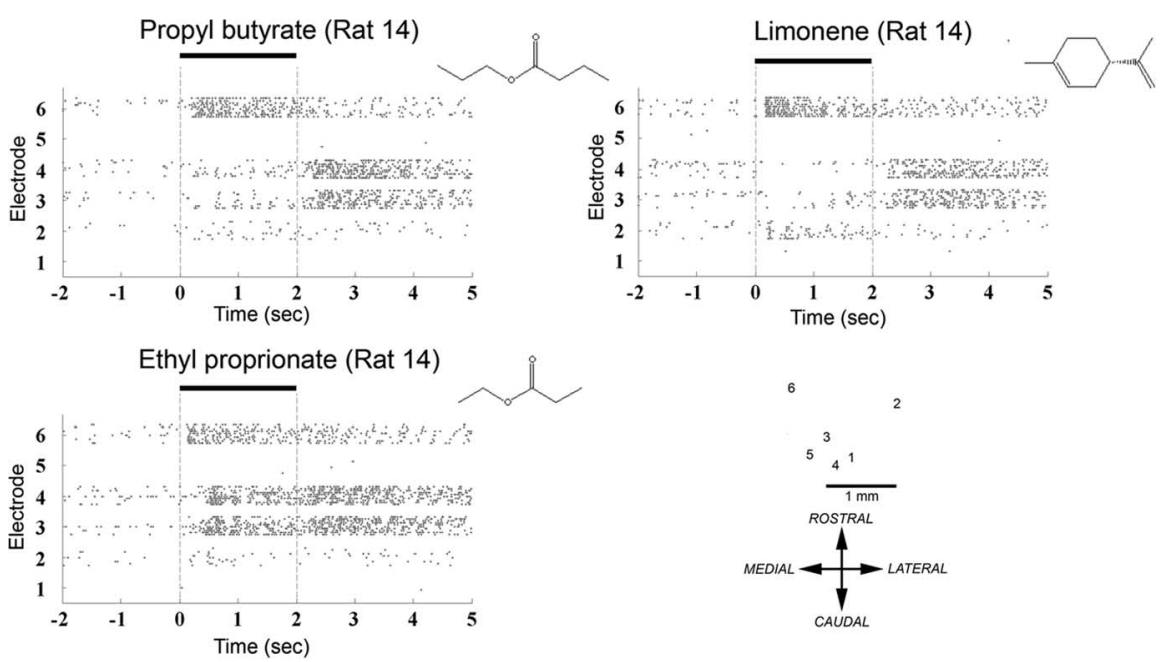

Figure 3. Individual odorants activate multiple recording sites. Representative raster displays of activity from six recording sites in a single rat to three different odorant stimuli are shown. The bottom right shows the relative spatial distribution of the recording sites to each other and shows that individual sites could be $>1 \mathrm{~mm}$ apart within an animal.

trainment, also varied between odorants at individual sites. Individual sites could discriminate between both molecularly similar odorants (Fig. 2, Rat 209 electrode 3, Propyl butyrate and Ethyl proprionate) as well as molecularly diverse odorants (Fig. 2, Rat 14 electrode 4, Heptanal and Limonene). Some sites showed very similar responses to a broad range of odorants (Fig. 2, Rat 217).

Responses to individual odorants could be observed in widespread regions of the anterior piriform cortex. For example as shown in Figure 3, ethyl proprionate produced excitation in three of five electrodes in this array, with electrode tip locations extending over $1 \mathrm{~mm}$ in the rostrocaudal direction. The same three sites also were excited by the molecularly similar molecule propyl butyrate, although with substantially different temporal characteristics. Finally, these three sites also responded to the molecularly dissimilar odorant limonene, although with both sites 3 and 4 
showing suppression rather than excitation. Thus, individual odorants evoke activity across wide regions of the piriform cortex.

Recording sites in the electrode array implants were localized within each rat in a grid with the rostral pole of the olfactory tubercle as anterior zero reference point and the midline as the mediolateral zero reference point. This coordinate system allowed us to create composite images of odorant-evoked activity across all animals. Only those electrode sites histologically confirmed as falling within the piriform cortex were included in these composites. Unit activity was converted to a pseudocolor rate scale, and activity maps were drawn with the dorsoventral axis collapsed to create two-dimensional plots of spatiotemporal odorant-evoked activity within piriform cortex. Activity from an individual site was assigned to a $100 \mu \mathrm{m}^{2}$ location, and activity was collapsed across $200 \mathrm{~ms}$ time bins.

As shown in Figure 4, a single odorant evokes widespread activation throughout the anterior piriform cortex. It must be emphasized that each recording site is discrete, and thus these are not continuous spatial maps such as provided by immunohistochemical techniques (Zinyuk et al., 2001; Illig and Haberly, 2003; Zou et al., 2005). However, these images demonstrate widespread spike-train activation across the rostrocaudal extent of the anterior piriform cortex by individual odorants. Furthermore, as described in more detail below, these images allow visualization of how these spatial patterns change over time.

To quantify these differences in spatial patterns, the evoked activity of multiunit clusters (200 ms time bins) was compared across odorants ( 10 repetitions of each odorant) using $t$ tests, and significant inter-odorant differences $(p<0.01)$ were plotted. Figure 5 shows examples of differences between patterns evoked by three odorants with approximately similar vapor pressures: isoamyl acetate (vapor pressure of $5 \mathrm{mmHg}$ ), propyl butyrate (vapor pressure of $6 \mathrm{mmHg}$ ), and limonene (vapor pressure of 2 $\mathrm{mmHg}$ ). Isoamyl acetate evoked significantly different activity in widespread regions of the anterior piriform cortex compared with both limonene and propyl butyrate at early $(800 \mathrm{~ms})$ stages of stimulation. By $1600 \mathrm{~ms}$, the differences between isoamyl acetate and propyl butyrate were less pronounced. The differences in activity evoked by isoamyl acetate and propyl butyrate reemerged at odorant offset. Propyl butyrate and limonene similarly produced distinct patterns of activity initially, but these rapidly diminished with time. Thus, although odorant-evoked activity may have lasted the entire duration of a stimulus presentation, the most odorant-distinctive ensemble activity pattern occurred during the initial phase (see below).

Odorant evoked activity was not only distributed over large regions of the anterior piriform cortex but also varied on smaller spatial scales. Analyses of single-unit activity recorded from single microelectrodes (in different animals from the electrode arrays) demonstrated that neighboring neurons did not necessarily share similar odorant receptive fields. Thirty-nine single-unit pairs were recorded, with 13 pairs tested extensively with two odorants, giving a total comparison set of 52. In 18 of 52 cases, both units of the pair responded to a given odorant, whereas in nearly half of the cases (23 of 52), one cell responded and the other did not. In the remaining 12 of 52 pairs, neither cell responded to the test odorants. In a single case, one cell was excited and the other suppressed to the same odorant. Thus, at both small and large spatial scales, odorant responses are distributed in piriform cortex.

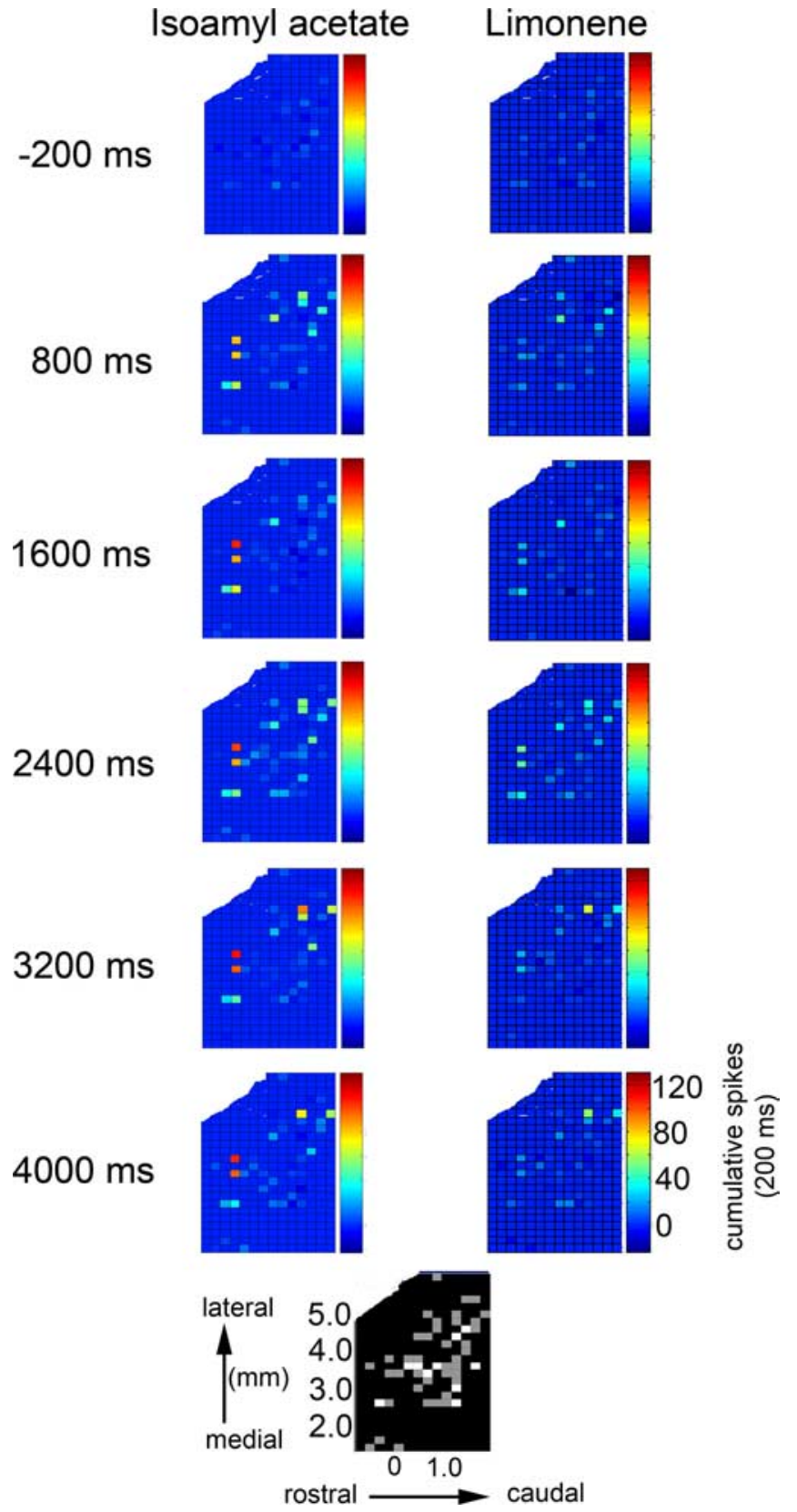

Figure 4. Individual odorants evoked unit activity distributed throughout the anterior piriform cortex. The images represent a view of the piriform cortex from the dorsal surface of the brain, with rostral to the left and the lateral edge of the brain to the top of each image. Electrode sites are plotted in the bottom in gray. White blocks signify that electrodes from two rats were located in that space. Color panels, Pseudocolor representations of multiunit activity at 59 recording sites plotted as a function of rostrocaudal and mediolateral location of the electrode site. Color represents cumulative activity in a $200 \mathrm{~ms}$ time bin over 10 repeats of the stimulus. Time is milliseconds from odorant onset (seconds stimulus duration). Both odorants evoked widespread activation of the anterior piriform cortex, although activity at specific recording sites varied with time.

\section{Temporal patterns}

The temporal pattern of odorant-evoked activity varied for individual recording sites across odorants and between sites. Latency to peak responses and the presence or absence of activity at odorant offset were the most robust variant response factors. For example, in Figure 2, responses included short-latency onset and prolonged excitation (e.g., Rat 209, response to ethyl proprionate) and long-latency onset (or stimulus offset-induced-) excita- 
tion (e.g., Rat 14, response to propyl butyrate). Variation in response latency could be expressed at a single site in an odorant-dependent manner (Fig. 2) and across sites in response to the same odorant (Fig. 3). Several sites in different animals showed what appeared to be odorant offset responses (Figs. 2, 3, Rat 14). This can also be observed in the plots of spatial activity patterns (Fig. 4).

As described above, the pattern of activity at each recording site could be compared between different odorants (Fig. 5). To quantitatively examine how the evoked activity of the ensemble of recordings varied over time, the number of sites that statistically differed between two odorants at a particular time point (200 ms bins) was measured for 15 different pairs of odorants (all possible combinations of isoamyl acetate, benzyl acetate, propyl butyrate, ethyl proprionate, heptane, and limonene). The distinctiveness of ensemble encoding of the two odorants increases as the number of significantly different sites increases. As shown in Figure 6A, using $200 \mathrm{~ms}$ time bins, the mean number of recording sites with significantly different activity rates between the two odorants peaked at $400 \mathrm{~ms}$ after odorant onset. This delay corresponds to the average respiratory cycle duration in anesthetized rats, and thus the maximal distinctiveness of piriform cortex ensemble encoding of odorants occurs during the first inhalation. The number of recording sites with activity rates significantly different between the two odorants then decreased over the course of the $2 \mathrm{~s}$ stimulus but showed a rebound at odorant offset.

Given the data demonstrating that animals can behavioral discriminate odors within $200 \mathrm{~ms}$ (Uchida and Mainen, 2003; Abraham et al., 2004), we further analyzed the peristimulus onset period at a higher temporal resolution ( $50 \mathrm{~ms}$ bins). As shown in Figure $6 B$, an increase in pattern distinctiveness emerged during the initial $100 \mathrm{~ms}$ of the first inhalation of the odorant (average respiratory waveform across all animals and stimuli is displayed, odorant onset triggered on exhalation-inhalation transition). Error bars in Figure $6 \mathrm{~B}$ are $95 \%$ confidence limits. Pattern distinctiveness was reduced during the initial exhalation but then rapidly strengthened on the next inhalation. Thus, sufficient odorant-specific information is present in the spatiotemporal patterns of piriform cortical activity to account for rapid behavioral odor discrimination.

In addition to temporal patterns in odorant-evoked activity, phasic activity related to the respiratory cycle was prominent in most single units as has been reported previously (Wilson, 1998; Litaudon et al., 2003). The most direct explanation for this respiration-locked activity is a reflection of similar activity in the cortical afferent, mitral/tufted cells (Buonviso et al., 1992; Chaput et al., 1992). Similar to the disparity in odor-evoked responses of nearby single units described above, paired recordings of neighboring single units revealed that neighboring cortical cells often fired at different phases of the respiratory cycle, in some cases nearly out-of-phase with each other (see Fig. 9). A plot of the mean vectors of respiration entrained activity in neighbor- ing cell pairs (Fig. 7) shows a poor correlation between the respiratory phase relationship of activity in neighboring single units (Pearson's $r=0.20 ; n=39$ single-unit pairs).

\section{Cell-cell interactions}

Cross-correlation analyses of paired single-unit recordings from single microelectrodes (not from the array recordings) were used to examine cell-cell interactions. Because of the low levels of spontaneous background activity in most cells, crosscorrelograms were determined from spike trains that included both spontaneous and odorant-evoked activity. In these initial analyses, no attempt was made to specifically compare correlated activity under different odorant stimulation conditions (see Discussion). In raw cross-correlations, 33 of 39 pairs (85\%) showed a significant $(p<0.01)$ (see Materials and Methods) peak in the cross-correlogram. To help isolate the effects of intrinsic from afferent activity in driving this correlated activity, the spike trains were shuffled by one respiratory cycle. Cross-correlograms were then determined from the shuffled spike trains, which were indicative of the patterning imposed on cortical neurons by the olfactory bulb and respiratory activity. These shuffled correlograms were then subtracted from the raw cross-correlograms to produce a difference cross-correlogram. Peaks in this final correlogram may reflect either direct cell-cell interactions or other, nonrespiration-related joint inputs to the cortical neurons. Of particular interest were off-center, short-latency (5-15 ms), narrow $(20-75 \mathrm{~ms})$ peaks, which may reflect direct synaptic interaction between the recorded cell pair (Perkel et al., 1967; Katz et al., 2002).

Subtraction of the respiration-shuffled cross-correlograms from the raw correlograms resulted in 26 of 39 cell pairs $(67 \%)$ still showing a significant peak in the difference crosscorrelogram. Of these, most (22 of 26) were broadly centered, 

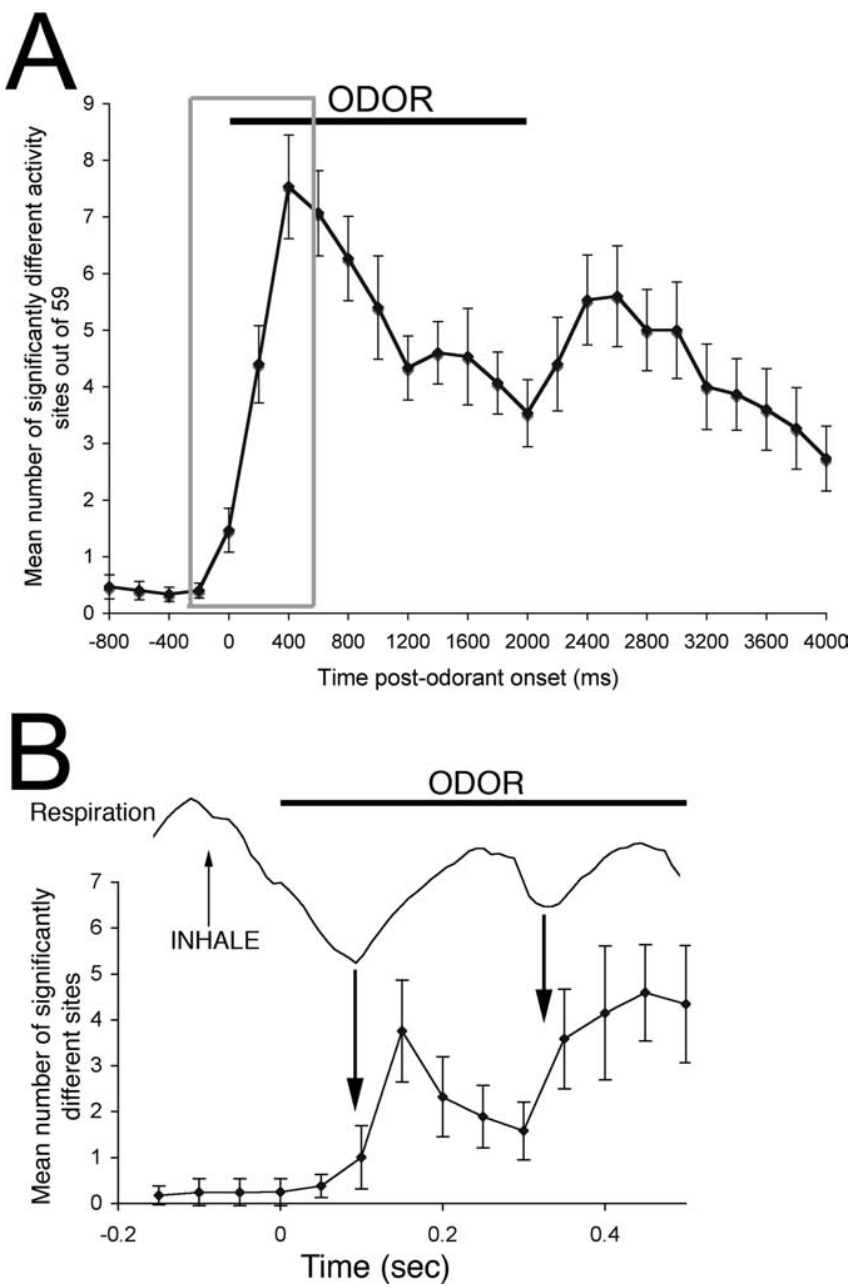

Figure 6. A, Mean number of recording sites showing a significant difference in activity evoked by two different odorant stimuli ( $n=15$ different odorant pair combinations; $200 \mathrm{~ms}$ bin width). The ensemble activity displayed the greatest difference between odorants during the first inhalation (400 ms). A second peak occurred at odorant offset. Odorant present during the horizontal bar. Mean \pm SEM. $\boldsymbol{B}$, Higher-resolution analysis (50 ms bins) of initial odor response with average respiratory waveform (chest wall movement) across all animals and stimuli displayed for alignment. Odorant onset in all cases occurred at the transition from inhalation to exhalation. An increase in pattern difference emerged nearly as the first inhalation began and peaked within $100 \mathrm{~ms}$. Mean $\pm 95 \%$ confidence limits.

similar to that shown in Figure 8. A relationship of this type may reflect joint excitatory inputs to the cell pair, although presumably not primarily respiration dependent given the shuffling protocol.

However, 4 of 26 cell pairs (15\% of cell pairs with significant cross-correlations) demonstrated a short-latency narrow offcenter peak in the difference cross-correlogram, similar to that shown in Figure 9. This type of correlogram is consistent with a potentially direct synaptic interaction between the two recorded cells. As described in Materials and Methods, to enhance the power of cross-correlation analyses in these slow-firing cortical neurons, spontaneous and odorant-evoked activity were combined. Spike trains from these four pairs, however, were further analyzed with a specific focus on spontaneous activity (activity occurring between stimulus presentations). Using Poisson-based statistical analyses described by Abeles (1982) and Bastian and Nguyenkim (2001) on the unsmoothed correlograms (1 ms bins), all four pairs showed significant $(p<0.05)$ off-center peaks at short latency during unstimulated periods.

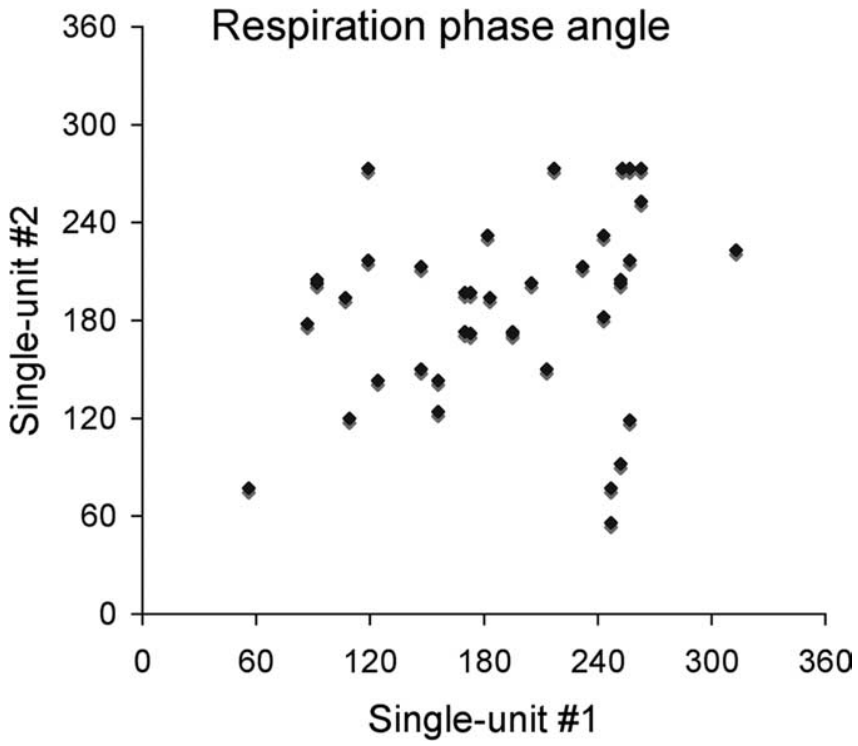

Figure 7. Neighboring single units did not preferentially fire in-phase with each other over the respiratory cycle. The correlation between the mean phase angle for simultaneously recorded single-unit pairs was 0.20 .

\section{Discussion}

The present results confirm and extend recent histological analyses demonstrating distributed odorant-evoked activity within the anterior piriform cortex (Illig and Haberly, 2003; Zou et al., 2005). Odorant stimulation evoked activity in multiunit clusters distributed over widespread regions of the anterior piriform cortex and individual clusters responded to multiple odorants. These results, together with previous work showing that individual single units in the piriform cortex are responsive to molecularly diverse odorants (Wilson, 2003; Kadohisa and Wilson, 2006a), are consistent with an ensemble coding of stimulus identity. The patterns of activity varied over time during and after a $2 \mathrm{~s}$ odor pulse. Both multiunit clusters and individual single units varied in their response onset latency, response duration, and entrainment to the respiration cycle. These results are consistent with a temporal dimension to the ensemble code. Maximal differentiation of ensemble activity to two different odorants occurred during the first inhalation and then decreased over the $2 \mathrm{~s}$ stimulus with a rebound at odorant offset. Finally, evidence of cell-cell intracortical connectivity was observed, emphasizing the importance of intrinsic association fiber connections in shaping cortical odorant responses, in addition to the role of afferent input from the olfactory bulb.

\section{Distributed activity}

Individual mitral cells terminate in the anterior piriform cortex in broad patches (Buonviso et al., 1991), with terminals of mitral cells conveying information from different olfactory receptors overlapping (Zou et al., 2001). Cortical pyramidal cells activated by a particular afferent input in turn further expand the population of responsive neurons through their intracortical association connections, with an individual piriform cortical neuron synapsing on $>1000$ other piriform cortical neurons (Johnson et al., 2000 ). Based on this anatomy, computational models of piriform cortex predict that many neurons will be responsive to a given odorant and that those responsive neurons will be broadly dispersed (Hasselmo et al., 1990; Haberly, 2001). The present results support that prediction. Furthermore, not only do odorants ac- 
tivate neurons scattered globally throughout the piriform cortex, on a local scale, neighboring single neurons also vary in their odorant response patterns. That is, when one of two neighboring single units responded to an odorant, the other unit also responded less than half of the time (43\%).

The present data do not provide sufficient resolution to detect evidence of spatial coding in piriform cortex, i.e., regions of cortex that may be differentially responsive to, and necessary for, coding of specific odorants. Immunohistochemical techniques suggest that such patterning may occur (Zou et al., 2005). The present findings of a strong temporal dimension to ensemble activity, however, suggests that these odorant-specific spatial activity patterns may change over the course of a 2 s odorant exposure.

Intracortical association fiber axons terminate on the proximal half of the pyramidal cell apical dendrite, providing a strong source of cell-cell excitation within piriform cortex (Neville and Haberly, 2004). The present results provide the first physiological evidence of which we are aware for association fiber connectivity modulating piriform cortex single-unit activity. Cross-correlogram analyses found that $15 \%$ of neighboring single-unit pairs with a significant correlation in activity showed a short-latency, narrowduration peak that is suggestive of direct cell-cell synaptic interaction (Perkel et al., 1967; Katz et al., 2002). These intracortical connections could significantly expand the size of the cortical ensemble responsive to a given odorant, beyond those cells directly contacted by excited mitral cells, which adds an important caveat in interpretation of odorant-evoked spatial patterns in piriform cortical activity. That is, the pattern of piriform cortical neurons responsive to a particular odorant reflects not only the pattern of input from the lateral olfactory tract but also the pattern and strength of intracortical connections. Although not directly tested here, it is hypothesized that these cell-cell interactions will also be odorant specific, with cell pairs communicating during stimulation with a subset of odorant stimuli and not during others. More data will be required to address this hypothesis.

Intracortical association fiber plasticity has also been hypothesized to underlie cortical memory for familiar odorants (Hasselmo et al., 1990; Haberly, 2001). Both behavioral (Rabin, 1988; Fletcher and Wilson, 2002) and cortical (Wilson, 2003; Kadohisa and Wilson, 2006a) odorant discrimination improves with familiarity and learning. Intracortical fibers serve as associative and feedback connections and express use-dependent plasticity such as long-term potentiation (Kanter and Haberly, 1990), and disruption of normal associative fiber plasticity impairs odor memory and perceptual learning (Hasselmo and Bower, 1992; Patil et al., 1998; Wilson, 2001; Fletcher and Wilson, 2002). The current data provide another method for examining how these intracor- tical connections are affected by experience. For example, the observed $15 \%$ interconnectivity of neighboring cell pairs may be expected to change as intracortical synaptic connections are modified with learning. The strength of cell-cell connections within cortical ensembles plays a critical role in overall ensemble function and the ability of the ensemble to recognize degraded inputs.

\section{Activity is distributed in both space and time}

Cortical ensemble activity was not only distributed in space across the anterior piriform cortex but also in time. Differences in activity evoked by separate odorants were most pronounced during the early phases of stimulation, peaking during the first inhalation (Fig. 6). Evoked-activity may have remained over the course of odorant stimulation, but patterns distinctive to each odorant faded, a finding consistent with other olfactory systems (Mazor and Laurent, 2005). Thus, the greatest information discriminating different odorants was present during the initial odorant onset, a finding that corresponds with behavioral evidence that rodents make even difficult odor discriminations within a single inhalation (Uchida and Mainen, 2003; Abraham et 


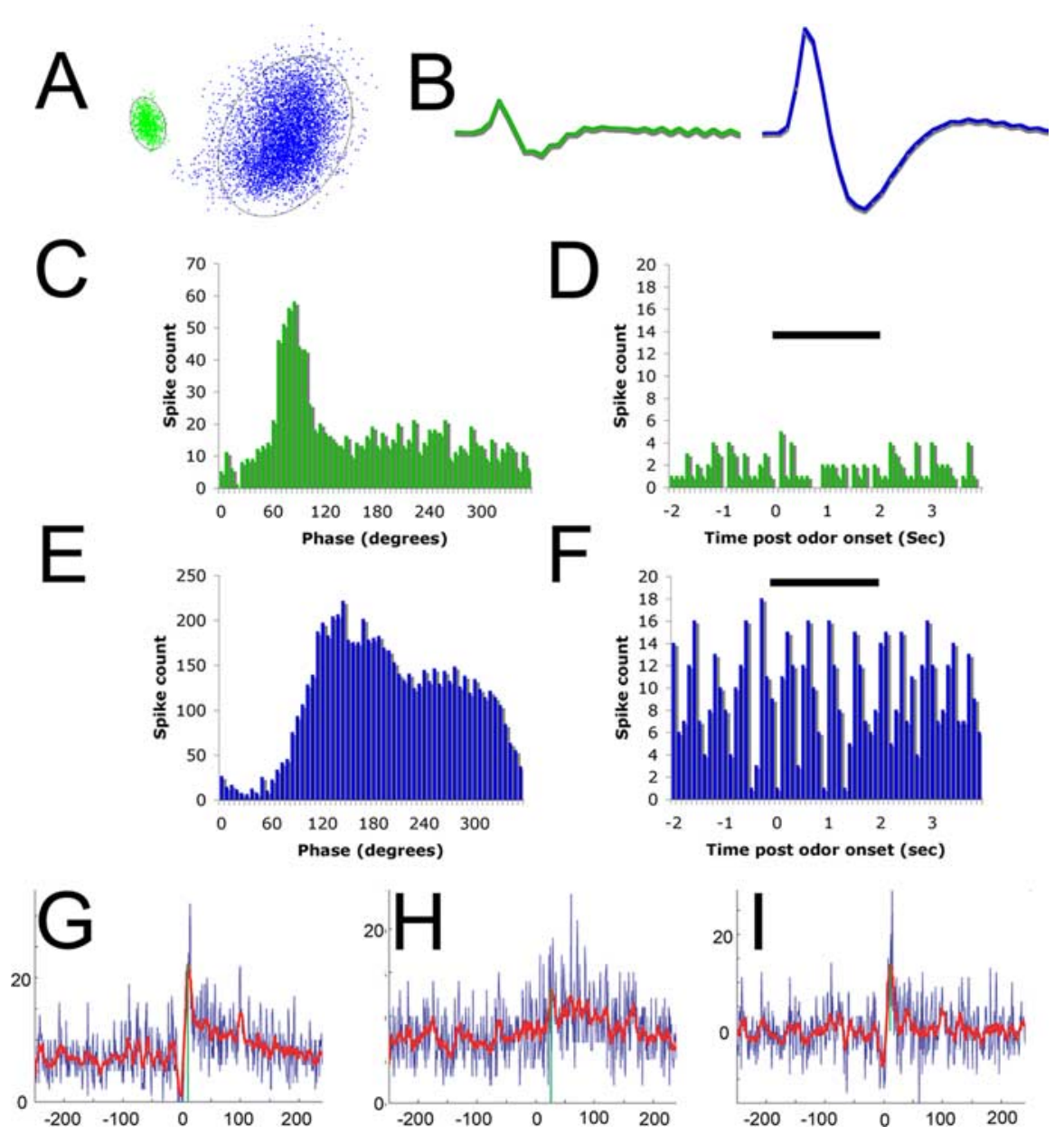

Figure 9. Representative example of a simultaneously recorded single-unit pair in the anterior piriform cortex. Details are as described in Figure 8. In this cell pair, the two cells fired out-of-phase with each other over the course of the respiratory cycle $(\boldsymbol{C}, \boldsymbol{E})$, and neither showed a significant response to odorant $(\boldsymbol{D}, \boldsymbol{F})$. The cross-correlogram shows a short-latency narrow peak offset from zero, indicative of a potential direct synaptic connection between these two neurons.

al., 2004; Uchida et al., 2006). Recording sites varied in their latency and duration of responses, and single sites varied in temporal response characteristics across odorants. The temporal structure of cortical activity may reflect both the dynamic responses of olfactory bulb glomerular (Spors et al., 2006) and mitral cell (Friedrich and Laurent, 2004) responses to odorants, as well as additional computations occurring intracortically. For example, it is unclear whether the early divergence of response patterns and appearance of offset cortical responses observed here reflect activity of inhibitory interneurons and horizontal connections within the piriform cortex or rather reflect patterns of afferent activity. Additional analyses of this cortical temporal structure during active odorant sampling in awake animals is warranted.

Again, in addition to the global temporal patterns across the anterior piriform cortex, even neighboring single units contribute relatively independently to the temporal structure of odorant-evoked activity. Neighboring single units showed a very poor correlation in their phasic activity over the respiratory cycle, with many cell pairs firing out-of-phase with each other during respiration. Similar independence has been reported in neighboring mitral cells (Egana et al., 2005), although in cells hypothesized to share a common glomerulus, activity may be more temporally linked (Buonviso et al., 1992).

\section{Summary}

Odorants evoke activity in a spatially scattered ensemble of neurons within anterior piriform cortex, and the ensemble activity includes a rich temporal structure. Ensemble activity is most distinctive between different odorants during the first inhalation, although it may show offset responses. The distributed spatial and temporal structure of cortical activity is present at both global and local scales, with neighboring single units contributing to encoding of different odorants and active at different phases of the respiratory cycle. Finally, cortical unit activity reflects not only afferent input from the olfactory bulb but also intrinsic activity within the intracortical association fiber system. These results provide important information as to how the spatiotemporal patterns of olfactory bulb output are read by cortical ensembles.

\section{References}

Abeles M (1982) Quantification, smoothing, and confidence limits for single-units' histograms. J Neurosci Methods 5:317-325.

Abraham NM, Spors H, Carleton A, Margrie TW, Kuner T, Schaefer AT (2004) Maintaining accuracy at the expense of speed: stimulus similarity defines odor discrimination time in mice. Neuron 44:865-876.

Alonso JM, Swadlow HA (2005) Thalamocortical specificity and the synthesis of sensory cortical receptive fields. J Neurophysiol 94:26-32.

Bastian J, Nguyenkim J (2001) Dendritic modulation of burst-like firing in sensory neurons. J Neurophysiol 85:10-22.

Buck LB (1996) Information coding in the vertebrate olfactory system. Annu Rev Neurosci 19:517-544.

Buonviso N, Revial MF, Jourdan F (1991) The Projections of mitral cells from small local regions of the olfactory bulb: an anterograde tracing study using PHA-L (Phaseolus vulgaris leucoagglutinin). Eur J Neurosci 3:493-500.

Buonviso N, Chaput MA, Berthommier F (1992) Temporal pattern analyses in pairs of neighboring mitral cells. J Neurophysiol 68:417-424.

Cang J, Isaacson JS (2003) In vivo whole-cell recording of odor-evoked synaptic transmission in the rat olfactory bulb. J Neurosci 23:4108-4116.

Chaput MA, Buonviso N, Berthommier F (1992) Temporal patterns in spontaneous and odour-evoked mitral cell discharges recorded in anaesthetized freely breathing animals. Eur J Neurosci 4:813-822.

Egana JI, Aylwin ML, Maldonado PE (2005) Odor response properties of neighboring mitral/tufted cells in the rat olfactory bulb. Neuroscience 134:1069-1080.

Fletcher ML, Wilson DA (2002) Experience modifies olfactory acuity: acetylcholine-dependent learning decreases behavioral generalization between similar odorants. J Neurosci 22:RC201(1-5).

Friedrich RW, Laurent G (2004) Dynamics of olfactory bulb input and output activity during odor stimulation in zebrafish. J Neurophysiol 91:2658-2669.

Gerstein GL, Perkel DH (1972) Mutual temporal relationships among neuronal spike trains. Statistical techniques for display and analysis. Biophys J 12:453-473.

Ghose GM (2004) Learning in mammalian sensory cortex. Curr Opin Neurobiol 14:513-518.

Gilbert CD, Sigman M, Crist RE (2001) The neural basis of perceptual learning. Neuron 31:681-697.

Haberly LB (2001) Parallel-distributed processing in olfactory cortex: new 
insights from morphological and physiological analysis of neuronal circuitry. Chem Senses 26:551-576.

Haberly LB, Bower JM (1989) Olfactory cortex: model circuit for study of associative memory? Trends Neurosci 12:258-264.

Hasselmo ME, Bower JM (1992) Cholinergic suppression specific to intrinsic not afferent fiber synapses in rat piriform (olfactory) cortex. J Neurophysiol 67:1222-1229.

Hasselmo ME, Wilson MA, Anderson BP, Bower JM (1990) Associative memory function in piriform (olfactory) cortex: computational modeling and neuropharmacology. Cold Spring Harb Symp Quant Biol 55:599-610.

Illig KR, Haberly LB (2003) Odor-evoked activity is spatially distributed in piriform cortex. J Comp Neurol 457:361-373.

Joerges J, Kuttner A, Galizia CG, Menzel R (1997) Representation of odours and odour mixtures visualized in the honeybee brain. Nature 387:285-288.

Johnson DM, Illig KR, Behan M, Haberly LB (2000) New features of connectivity in piriform cortex visualized by intracellular injection of pyramidal cells suggest that "primary" olfactory cortex functions like "association" cortex in other sensory systems. J Neurosci 20:6974-6982.

Kadohisa M, Wilson DA (2006a) Separate encoding of identity and similarity of complex familiar odors in piriform cortex. Proc Natl Acad Sci USA 103:15206-15211.

Kadohisa M, Wilson DA (2006b) Olfactory cortical adaptation facilitates detection of odors against background. J Neurophysiol 95:1888-1896.

Kanter ED, Haberly LB (1990) NMDA-dependent induction of long-term potentiation in afferent and association fiber systems of piriform cortex in vitro. Brain Res 525:175-179.

Katz DB, Simon SA, Nicolelis MA (2002) Taste-specific neuronal ensembles in the gustatory cortex of awake rats. J Neurosci 22:1850-1857.

Leon M, Johnson BA (2003) Olfactory coding in the mammalian olfactory bulb. Brain Res Brain Res Rev 42:23-32.

Lin DY, Shea SD, Katz LC (2006) Representation of natural stimuli in the rodent main olfactory bulb. Neuron 50:937-949.

Litaudon P, Amat C, Bertrand B, Vigouroux M, Buonviso N (2003) Piriform cortex functional heterogeneity revealed by cellular responses to odours. Eur J Neurosci 17:2457-2461.

Mazor O, Laurent G (2005) Transient dynamics versus fixed points in odor representations by locust antennal lobe projection neurons. Neuron 48:661-673.

Meredith M (1986) Patterned response to odor in mammalian olfactory bulb: the influence of intensity. J Neurophysiol 56:572-597.

Neville KR, Haberly L (2004) Olfactory cortex. In: The synaptic organiza- tion of the brain, Ed 5 (Shepherd GM, ed), pp 415-454. New York: Oxford UP.

Patil MM, Linster C, Lubenov E, Hasselmo ME (1998) Cholinergic agonist carbachol enables associative long-term potentiation in piriform cortex slices. J Neurophysiol 80:2467-2474.

Perkel DH, Gerstein GL, Moore GP (1967) Neuronal spike trains and stochastic point processes. II. Simultaneous spike trains. Biophys J $7: 419-440$

Rabin MD (1988) Experience facilitates olfactory quality discrimination. Percept Psychophys 44:532-540.

Rennaker RL, Ruyle AM, Street SE, Sloan AM (2005) An economical multichannel cortical electrode array for extended periods of recording during behavior. J Neurosci Methods 142:97-105.

Spors H, Wachowiak M, Cohen LB, Friedrich RW (2006) Temporal dynamics and latency patterns of receptor neuron input to the olfactory bulb. J Neurosci 26:1247-1259.

Tabor R, Yaksi E, Weislogel JM, Friedrich RW (2004) Processing of odor mixtures in the zebrafish olfactory bulb. J Neurosci 24:6611-6620.

Uchida N, Mainen ZF (2003) Speed and accuracy of olfactory discrimination in the rat. Nat Neurosci 6:1224-1229.

Uchida N, Kepecs A, Mainen ZF (2006) Seeing at a glance, smelling in a whiff: rapid forms of perceptual decision making. Nat Rev Neurosci 7:485-491.

Vickers NJ, Christensen TA, Hildebrand JG (1998) Combinatorial odor discrimination in the brain: attractive and antagonist odor blends are represented in distinct combinations of uniquely identifiable glomeruli. J Comp Neurol 400:35-56.

Wilson DA (1998) Habituation of odor responses in the rat anterior piriform cortex. J Neurophysiol 79:1425-1440.

Wilson DA (2001) Scopolamine enhances generalization between odor representations in rat olfactory cortex. Learn Mem 8:279-285.

Wilson DA (2003) Rapid, experience-induced enhancement in odorant discrimination by anterior piriform cortex neurons. J Neurophysiol 90:65-72.

Wilson DA, Stevenson RJ (2003) The fundamental role of memory in olfactory perception. Trends Neurosci 26:243-247.

Zinyuk LE, Datiche F, Cattarelli M (2001) Cell activity in the anterior piriform cortex during an olfactory learning in the rat. Behav Brain Res 124:29-32.

Zou Z, Horowitz LF, Montmayeur JP, Snapper S, Buck LB (2001) Genetic tracing reveals a stereotyped sensory map in the olfactory cortex. Nature 414:173-179.

Zou Z, Fusheng L, Buck LB (2005) Odor maps in the olfactory cortex. Proc Natl Acad Sci USA 102:7724-7729. 